University of Nebraska - Lincoln

DigitalCommons@University of Nebraska - Lincoln

Industrial and Management Systems

Engineering Faculty Publications

Industrial and Management Systems

Engineering

2004

\title{
Human performance reliability: On-line assessment using fuzzy logic
}

William J. Kolarik

Oklahoma State University, kolarik@okstate.edu

Jeffrey C. Woldstad

University of Nebraska-Lincoln, Jeffrey.Woldstad@sdsmt.edu

Susan Lu

SUNY Binghamton, slu@binghamton.edu

Huitian Lu

South Dakota State University, huitianlu@sdstate.edu

Follow this and additional works at: https://digitalcommons.unl.edu/imsefacpub

Part of the Operations Research, Systems Engineering and Industrial Engineering Commons

Kolarik, William J.; Woldstad, Jeffrey C.; Lu, Susan; and Lu, Huitian, "Human performance reliability: On-line assessment using fuzzy logic" (2004). Industrial and Management Systems Engineering Faculty Publications. 12.

https://digitalcommons.unl.edu/imsefacpub/12

This Article is brought to you for free and open access by the Industrial and Management Systems Engineering at DigitalCommons@University of Nebraska - Lincoln. It has been accepted for inclusion in Industrial and Management Systems Engineering Faculty Publications by an authorized administrator of DigitalCommons@University of Nebraska - Lincoln. 


\title{
Human performance reliability: On-line assessment using fuzzy logic
}

\author{
William J. Kolarik, ${ }^{1}$ Jeffrey C. Woldstad, ${ }^{2}$ Susan Lu, ${ }^{3}$ And Huitian Lu ${ }^{4}$
}

1 School of Industrial Engineering and Management, College of Engineering, Oklahoma State University, Stillwater, OK 74078, USA; email kolarik@okstate.edu

2 Department of Industrial Engineering, Texas Tech University, Lubbock, TX 79409, USA; email jeff.woldstad@coe.ttu.edu

3 Department of Systems Science and Industrial Engineering, SUNY Binghamton, NY 13902, USA; email: slu@binghamton.edu

4 Department of Engineering Technology and Management, College of Engineering, South Dakota State University, Brookings, SD 57007, USA; email huitianlu@sdstate.edu

Corresponding author - W. J. Kolarik

\begin{abstract}
This paper presents an on-line approach to monitoring human performance in terms of conditional reliability when one is performing a task. Unlike traditional human reliability analysis, this approach develops a dynamic model that is able to cope with constantly changing conditions that affect operator performance. A fuzzy knowledge-based assessment approach is developed in order to deal with uncertainty and subjectivity associated with human performance assessment. This technology includes three main parts/functions: (i) on-line performance monitoring; (ii) real-time performance forecasting; and (iii) performance reliability assessment. The technology is demonstrated in real-time and provides timely conditioned reliability information regarding task success/failure. In general, this technology offers human reliability assessment under highly dynamic circumstances.
\end{abstract}

\section{Introduction}

Physiological, psychological and physical factors all influence human reliability. Over the years, many approaches to human reliability assessment have been proposed (Swain and Guttmann, 1983; Embrey et al., 1984; Hannaman et al., 1985; Weston et al., 1987). Most approaches estimate the probability of human error. Estimations are usually derived from historical data and/or expert judgment, under static conditions.

In many cases, human operators may be required to work in dynamic situations where conditions are continuously changing over time. In addition, individuals performing a particular task vary widely in capabilities and in their response to external situations (Kolarik, Woldstad, Lu and Lu, 1998; Kolarik, Woldstad and Lu, 2000).

In this paper, a new approach to human reliability prediction is developed to accommodate the dynamic nature of human performance. Human performance is assessed in real-time based on past, present and forecasted performance with reference to given failure criteria. This realtime human reliability model consists of three major functions: (i) on-line performance monitoring; (ii) real-time performance forecasting; and (iii) performance reliability assessment, which are discussed in Section 3. A specific method utilizing a fuzzy knowledge base to convert the forecasted performance to reliability estimates is described in Section 4. Key issues in implementation include performance measure identification, failure definitions and performance forecasting. Figure 1 shows the functional block diagram of the assessment model structure.

\section{Human performance reliability and prediction model}

\subsection{The concept of human performance reliability}

Human performance reliability is proposed in order to evaluate and predict an individual's performance under dynamic conditions when one is performing a task (Kolarik, Woldstad, Lu and Lu, 1998; Kolarik, Woldstad and Lu, 2000). Human performance reliability is defined as "the conditional probability that human performance measures/metrics are greater (or less) than given critical limits/thresholds for a given future period of time" (Kolarik, 1995; Kolarik, Woldstad, Lu and Lu, 1998; Lu, Kolarik and Lu, 2001). Human performance measures/ 


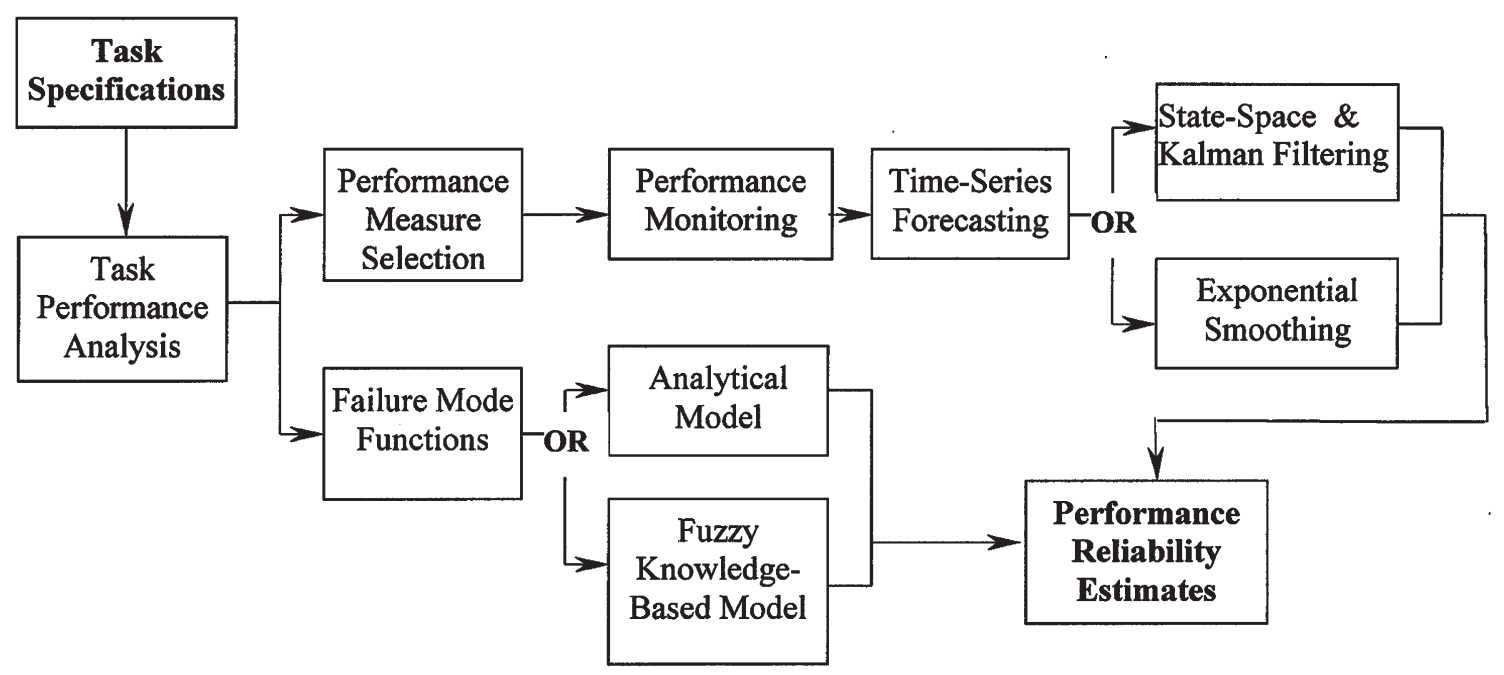

Figure 1. The functional block diagram of the assessment model structure.

metrics are physical variables/signals that are highly correlated with performance. The critical limits are clearly defined boundaries for the human performance measures/metrics that separate unacceptable performance from acceptable performance. Human performance reliability is essentially the extension of its physical counterpart (Lu, Lu and Kolarik, 2001).

In general, humans may exhibit different failure modes (errors) in performing a task. Each failure mode may be affected by several performance measures/metrics. Hence, multiple performance measures/metrics and multiple failure modes (errors) are a general scheme in model consideration (Lu, 1999). Suppose that there are $p$ performance measures and $m$ failure modes (errors) regarding a defined human task. Assume that each failure mode (error) is defined by a function in terms of performance measures/metrics $y_{1}, y_{2}, \ldots, y_{p}$ :

$$
s_{i}\left(y_{1}, y_{2}, \ldots, y_{p}\right)=0, \quad i=1,2, \ldots, m,
$$

where $s_{i}$ represents the failure mode function regarding failure mode $i$.

For failure mode (error) $i$, its conditional failure probability (for the smaller-is-better case where a system is defined as a failure when the performance measure exceeds an upper critical limit during time $\Delta t$ ) can be calculated by:

$$
F_{i}(\Delta t)=\int \cdots \iiint_{\Omega_{i}} f_{t}\left(y_{1}, y_{2}, \ldots, y_{p}\right) \mathrm{d} y_{1} \mathrm{~d} y_{2} \cdots \mathrm{d} y_{p} .
$$

Here, $f_{t}\left(y_{1}, y_{2}, \ldots, y_{p}\right)$ represents the joint probability density function of performance variables $y_{i}$ at time $t$. It can be obtained from a forecasted mean vector and covariance matrix associated with the performance measures. Assuming the joint statistical distribution is multivariate normal, $\Omega_{i}$ is the surface defined by failure mode definition function $s_{i}\left(y_{1}, y_{2}, \ldots, y_{p}\right)>0$.
The human performance reliability considering all failure (errors) modes is given by:

$R(\Delta t)=1-\int \cdots \iiint_{\Omega_{i}} f_{t}\left(y_{1}, y_{2}, \ldots, y_{p}\right) \mathrm{d} y_{1} \mathrm{~d} y_{2} \cdots \mathrm{d} y_{p}$

where $\Omega$ represents: $\Omega_{1} \cup \Omega_{2} \cup \ldots \cup \Omega_{m^{\prime}}$ which implies that any human failure mode will lead to the failure of the task.

For example, assume that there are two performance measures and two failure modes in a specific human task. Figure 2 shows a corresponding multivariate performance reliability concept. The ellipses are contours of probability density function $f_{t}\left(y_{1}, y_{2}\right)$. The two straight lines $S_{1}\left(y_{1}\right.$, $\left.y_{2}\right)$ and $S_{2}\left(y_{1}, y_{2}\right)$ are critical surfaces corresponding to failure modes 1 and 2. The integration of probability density function $f_{t}\left(y_{1}, y_{2}\right)$ over area $\Omega_{1}$ represents the failure probability regarding failure mode 1 . The integration of $f_{t}$ $\left(y_{1}, y_{2}\right)$ over area $\Omega_{2}$ represents the probability regarding failure mode 2 . The integration over the union of $\Omega_{1}$ and

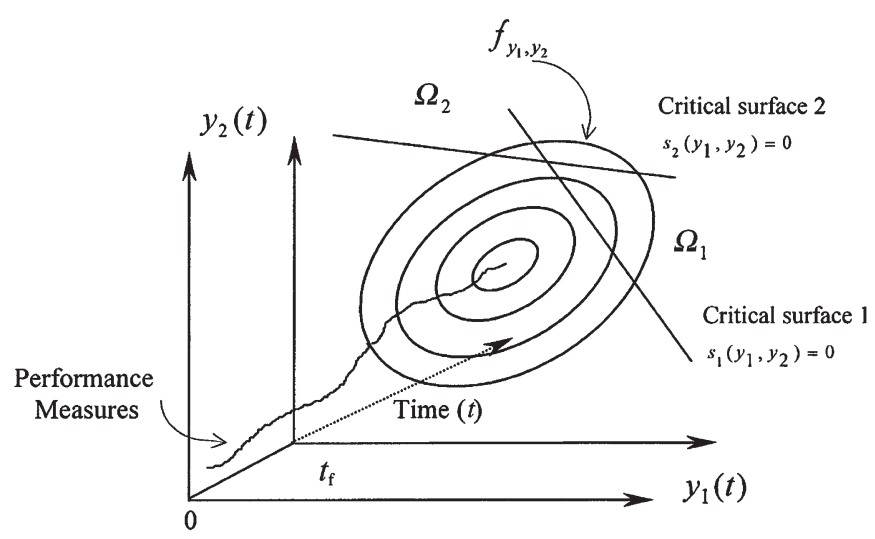

Figure 2. Human performance reliability concept (two performance measures and two failure modes). 
$\Omega_{2}$ represents the probability with respect to both failure modes; an overall human failure probability $(\mathrm{Lu}, 1999)$.

\subsection{Human performance reliability prediction model}

As discussed above, the implementation of human performance reliability is based on forecasted results of performance measures. Performance measures, monitored and sampled at specified intervals, serve as the input of the Human Performance Reliability Prediction (HPRP) model. They are a realization of a stochastic process and can be treated as a multivariate time-series (Lu, 1999). They are modeled and forecasted by multivariate timeseries forecasting methodologies. Assuming these variables/measures are multivariate-normal distributed, then the joint probability density function in Equation (1) can be obtained from the forecasted mean vector and covariance matrix. Human performance reliability is calculated by Equation (2), while the integration area is defined by the failure definition (discussed in Section 3).

HPRP models provide a means for human reliability assessment in real-time. Compared with traditional human reliability models, the proposed human performance reliability models differ in several critical respects (Lu, Lu and Kolarik, 2001): (i) each working individual is the subject of modeling; (ii) the model is implemented in real-time, using on-line sensors; (iii) the model is driven by a time-varying function that can accommodate continuously changing situations and/or environments; and (iv) the model can influence operational decisions in realtime. The HPRP model can be applied to human-task related reliability applications where human performance measures can be sampled and collected over time. A specific human-task related implementation example is described in Section 4. In practice, a full understanding of task requirements is a must for proper model application. In addition, the following facets should be considered for a specific application: (i) the selected performance variable/measures should be highly correlated with the human performance; (ii) these variables should be monitored over time; and (iii) the failure mode(s) function should be defined in terms of performance measure(s) either in a crisp or fuzzy form.

\section{HPRP model implementation}

Procedures for implementation of the HPRP model generally are: (i) understanding the task specification; (ii) analyzing possible human performance failures; (iii) identifying human performance measures; (iv) defining performance failures in terms of performance measures; (v) monitoring and modeling performance on-line; and (vi) predicting real- time performance reliability (Kolarik et al., 2000). Here, task specifications and requirements serve as the basis of failure definition. The following sections will discuss the key issues in the implementation of HPRP models.

\subsection{Human performance measures}

Physiological, psychophysical and physical factors impact on human performance. In addition to these factors, the situational conditions (external situation) of environment, equipment and human-machine interface influence human performance. The interactions between these factors complicate human reliability modeling and assessment (Kolarik, $\mathrm{Lu}$ and $\mathrm{Lu}, 1998$ ). Traditional means to consider the impact of these factors on human reliability include performance shaping factors (Embrey et al., 1984) in which the probability of human performance failure is modified according to tabulated values or expert (subjective) opinions.

In HPRP applications, performance parameters are monitored in real-time. Applications pose challenges for monitoring and modeling due to situational complexity and the resulting multiple dimensions in performance matrices. In some cases one selects indirect measures that are highly correlated with human performance. They are generally the resultant characteristics of human performance metrics (physiological, psychophysical and physical) and the work environment (environment, equipment and human- machine external interface), instead of causerelated metrics (those parameters that impact on human performance). For example, if accuracy in positioning operations is the main concern in a task, then position relevant performance parameters can be chosen as performance measures. Three basic criteria for the selection of performance metrics exist:

1. The performance metrics selected must characterize an important aspect of human performance for the task under study.

2. For each performance metric, there must be a clearly defined criterion (failure mode function) that separates unacceptable performance from acceptable performance.

3. Metrics must be measurable and monitored in real-time.

\subsection{Definition of human performance failure}

Task specifications and requirements serve as the basis for model application, calibration and failure definition. Two basic methods are applicable in failure definition: (i) the analytical method (clear-cut definition); and (ii) the intelligent method (fuzzy definition). In the analytical method, failure is defined in the form of mathematical functions in terms of performance measures, such as $s_{i}\left(y_{1}, y_{2}, \ldots, y_{p}\right)>0$, where $y_{1} \ldots y_{p}$ are performance measures. For each failure mode $i$, the function may include all the performance measures or part of them. Failure definition requires a careful analysis of the human task. Definition may involve off-line experimentation (and/or situation simulation) and statistical data analysis methods such as multivariate regression. From these analyses, failure surfaces are generated.

In the case that a clear-cut critical limit is difficult to obtain, an expert's knowledge about human performance failures can be incorporated in failure definitions. This 


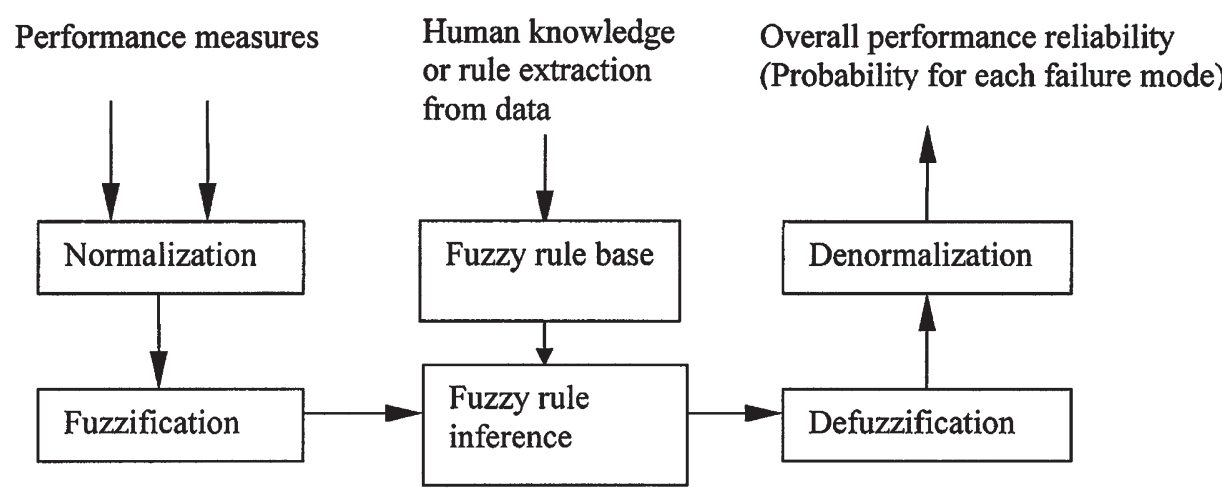

Figure 3. The structure of a fuzzy reliability estimator in the Multiple-Input-Multiple-Output (MIMO) case.

method is called fuzzy failure definition. In this method, human performance reliability regarding each failure mode is defined by a set of linguistic fuzzy: "if-then" rules based on experiences or knowledge of experts about failures. For example: "if performance variable one is extremely low and performance variable two is moderately high, then the probability of failure mode 1 may be extremely low and failure mode 2 moderately high." In cases such as these human performance reliabilities can be estimated by implementing corresponding fuzzy rules.

Fuzzy rules are stored and implemented in a fuzzy reliability estimator. A fuzzy reliability estimator may be viewed as a real-time expert system for performance reliability assessment. The core of a fuzzy reliability estimator is a linguistic description of conditional reliability under a given input performance state. The configuration of a fuzzy performance reliability estimator is depicted in Figure 3. The inputs to the estimator are performance measures forecast in real-time in the form of crisp values. The outputs of the estimator are the crisp values pertaining to overall human performance reliabilities, or separate performance reliabilities corresponding to different failure modes. The estimator usually includes six components: normalization and denormalization, fuzzification and defuzzification, fuzzy rule and rule inference (Hines, 1997; Driankov et al., 1999).

In Figure 3 normalization is first used to perform scale transformations in which the performance variables are mapped into a common (normalized) discourse of the same magnitude. Fuzzification is then applied to transfer normalized performance measures into linguistic variables (called fuzzy sets). In this transformation, a crisp value is represented by fuzzy sets together with their corresponding membership functions in a value between $[0,1]$, which indicates the degree to which a fuzzy variable belongs to the fuzzy set. In general, a crisp performance measure can be represented by different fuzzy sets with different membership functions. A fuzzy rule base is a database of fuzzy "if-then" rules. These fuzzy rules are built based on the knowledge about performance reliability from historical information and/or experience of experts. They are a set of linguistic relationships between the conditions of performance measures and the estimated reli- ability in the form of "if-then" rules. In rule inference, multiple fuzzy rules corresponding to definite performance measures are fired. The Degree of Fulfillment (DoF) value for each rule is implemented by "AND" fuzzy set operations and then used in the defuzzification step. Using defuzzification, all multiple fuzzy rules fired in the fuzzy rule inference are combined and converted to a crisp value. In this description, the technique of "center-of-mass" is applied in this step. After defuzzification, performance reliability is obtained as a normalized crisp value converted to its original universe of discourse $[0,1]$ by denormalization. This conversion serves as the output of the performance reliability estimator. By implementation of the fuzzy rules for each failure mode, the estimator can output a separate performance reliability for each failure mode and an overall performance reliability (considering all failure modes).

\subsection{Forecasting of performance measures in HPRP model}

Several forecasting technologies are available for multivariate time series: exponential smoothing, state-space modeling, neural network modeling, fuzzy time-series modeling and ARIMA modeling. Considering timeliness and accuracy, state-space modeling and exponential smoothing are feasible methods for real-time applications. State-space modeling has an advantage in accuracy for both one-step and multi-step (looking-ahead) forecasts over exponential smoothing; while exponential smoothing has an advantage in timeliness due to less matrix computation (Lu, 1999). In addition, it is possible to combine exponential smoothing with fuzzy performance reliability in cases where timeliness is critical and high precision estimates are not required.

\subsection{Multivariate exponential smoothing}

Exponential smoothing models utilize time-decreasing weighted averages. Success in applying exponential smoothing forecasting technology to physical system performance reliability assessment for the univariate case was reported by Lu (1998). Enn et al. (1982) introduced a multivariate exponential smoothing model, which is an extension of univariate adaptive exponential smoothing. 
Suppose a multivariate time series $\mathbf{y}_{t}\left(\in R^{p \times 1}\right)$ can be decomposed into a vector of permanent influences $\beta_{t}(\epsilon$ $R^{p \times 1}$ ) and a vector of disturbances having mean zero and covariance matrix $\Sigma_{\varepsilon}\left(\in R^{p \times p}\right)$ :

$$
\mathbf{y}_{t}=\beta_{t}+\varepsilon_{t}, \quad t=1,2, \ldots, n .
$$

Assume that the permanent component $\beta_{t}$ follows a firstorder Markov process:

$$
\beta_{t}=\beta_{t-1}+\eta_{t}
$$

where, $\eta_{t} \in R^{p \times 1}$ is a white noise vector with $E\left(\eta_{t}\right)=0$ and covariance matrix $\operatorname{Var}\left(\eta_{t}\right)=\Sigma_{\eta}$. Suppose $\hat{\mathbf{y}}_{t+1}$ represents the predicted vector of $\mathbf{y}_{t+1}$, based on the information available at an instant of time $t$ with minimum conditional mean-square error:

$$
\hat{\mathbf{y}}_{t+1}=E\left(\beta_{\mathrm{t}} \mid \mathbf{y}_{t}, \mathbf{y}_{t-1}, \ldots, \mathbf{y}_{1}\right) .
$$

The multivariate recursive equation for the one-step ahead forecast of the conditional mean of $\beta_{t}$ is:

$$
\hat{\beta}_{t}=\hat{\beta}_{t-1}+\alpha_{t}\left(\mathbf{y}_{t}-\hat{\beta}_{t-1}\right),
$$

where $\alpha_{t} \in R^{p \times p}$ is the smoothing matrix, which can be obtained in a recursive fashion, and

where

$$
\alpha_{t}=\left(\mathbf{S}_{t-1}+\lambda \Sigma\right)\left(\mathbf{S}_{t-1}+\Sigma\right)^{-1}
$$

and

$$
\lambda=\left|\Sigma_{\eta}\right| /|\Sigma|,
$$

$$
\Sigma=\Sigma_{\eta}+\Sigma_{\varepsilon}
$$

Here, $\lambda$ is called the allocation parameter. $S_{t-1}$ in Equation (7) is the conditional variance of $\beta_{t-1}$, given the observations $\mathbf{y}_{t}, \mathbf{y}_{t-1}, \ldots, \mathbf{y}_{1^{\prime}}$

$$
\mathbf{S}_{t-1}=\left(1-\alpha_{t-1}\right)\left(\mathbf{S}_{t-2}+\Sigma_{\eta}\right) .
$$

\subsection{State-space modeling and Kalman filtering}

The state-space model was first applied to time-series analysis in 1974 by Akaike. Since that time, many successful applications have been described (Harvey, 1990; Ng and Young, 1990; Young et al., 1991; Young, 1994). Using Kalman filtering, both single and multiple time series can be processed recursively in the estimation of states and time forecasting. In this application, time series are usually first decomposed into a structural time-series form such as trend, stochastic perturbation, periodic and white noise components. Each component is then identified and modeled in a state-space form separately. The complete state-space model can be obtained by combining all these separate models together (Young et al., 1991).

The general concept behind state-space modeling is that the time-series vector, $\mathbf{y}(t)$, is made up of observation variables that depend on a possibly unobserved state vector $\mathbf{x}(t)$. Here, the state vector $\mathbf{x}(t)$ is defined as a minimum set of information from the present and past such that the future behavior of the system can be completely described by the information about the present state and future inputs (Ogata, 1997). The state-space is represented by an observation equation and a transition equation:

$$
\begin{aligned}
& \mathbf{x}(t)=\mathbf{F x}(t-1)+\mathbf{G} \eta(t-1), \\
& \mathbf{y}(t)=\mathbf{H} \mathbf{x}(t)+\varepsilon(t),
\end{aligned}
$$

where, $\mathbf{x}(t)$ is a state vector; $\mathbf{H}, \mathbf{F}$, and $\mathbf{G}$ are parameter matrices (or vectors) of the state-space model; $\varepsilon(t)$ is a white noise vector; and $\eta(t)$ is a disturbance.

In performance failure prediction modeling, multivariate performance measures are first decomposed into the low- frequency trend vector $\mathbf{T}(t)$, periodical component $\mathrm{S}(\mathrm{t})$ and residual vector $\mathbf{P}(t)$. $\mathbf{T}(t)$ can be modeled by a multivariate random walk a multivariate integrated random walk or a multivariate smoothed random walk $(\mathrm{Ng}$ and Young, 1990). In the demonstration that follows, the Multivariate Integrated Random Walk (MIRW) is chosen to represent the local low-frequency trend. The integrated random walk has advantages in representing the local trend component (Harvey, 1990; Ng and Young, 1990).

Once a model has been written in a state-space form, Kalman filtering can be applied to make single-step or multi-step forecasts in a recursive manner. Kalman filtering can be written conveniently in a general "prediction-correction" form (Ng and Young, 1990; Young, 1994). One-step and multi-step forecasts are developed in the following equations:

For a one-step forecast:

$$
\begin{aligned}
\hat{\mathbf{x}}(t+1 \mid t) & =\mathbf{F} \hat{\mathbf{x}}(t), \\
\hat{\mathbf{y}}(t+1 \mid t) & =\mathbf{H} \hat{\mathbf{x}}(t+1 \mid \mathbf{t}) .
\end{aligned}
$$

For a multi-step forecast

$$
\begin{aligned}
& \hat{\mathbf{x}}(t+l \mid t)=\mathbf{F}^{l} \hat{\mathbf{x}}(t), \\
& \hat{\mathbf{y}}(t+l \mid t)=\mathbf{H} \hat{\mathbf{x}}(t+l \mid \mathbf{t}) .
\end{aligned}
$$

The $l$-step ahead prediction error can be evaluated as:

$$
\mathbf{e}(t+l \mid t)=\mathbf{y}(t+l)-\hat{\mathbf{y}}(t+l \mid t),
$$

and the variance of this forecast can be calculated as (Young et al., 1991):

$$
\operatorname{Var}\{\mathbf{e}(\mathrm{t}+1 \mid t)\}=\mathbf{R}\left[\mathbf{I}+\mathbf{H P}(t+l \mid t) \mathbf{H}^{\mathrm{T}}\right] .
$$

Once the mean value and covariance matrix of forecasted performance measures are obtained using the above equations, the probability density function in Equation (1) is then applied. 


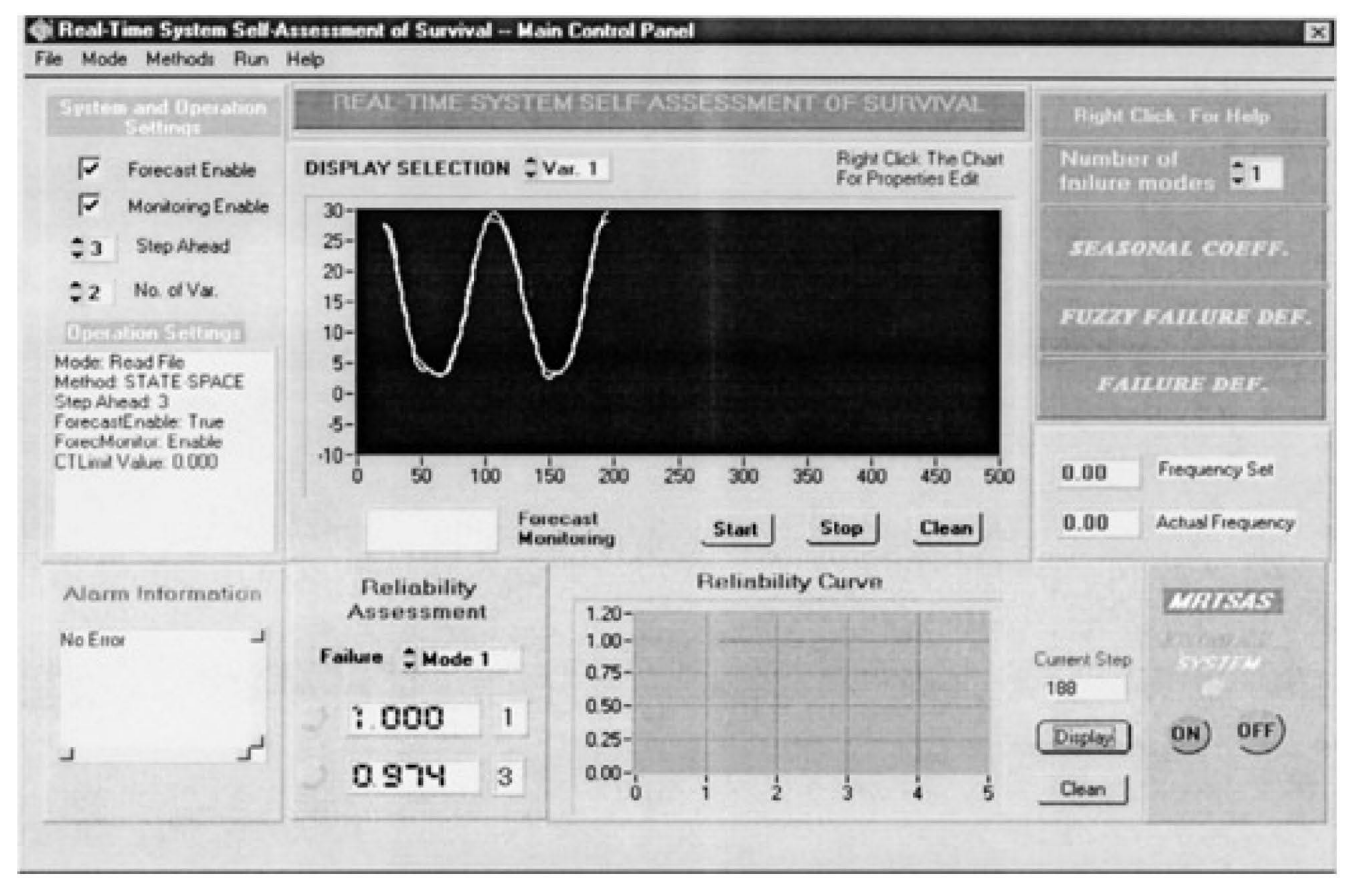

Figure 4 . The main interface panel of the prototype.

\section{Model verification}

Real-time application of the HPRP model requires the use of computer aids. A software prototype has been developed based on the performance reliability model. Figure 4 shows the main interface panel of the prototype. Through the main panel, the user can select options to set up parameters for a specific human task application. The operating setup option includes forecast steps, number of performance variables and number of failure modes, etc. Through the user interface, the failure mode definition method can be chosen (between crisp failure mode definition and fuzzy failure mode definition). The sampled performance measurements and one-step forecast results can be displayed in the chart "Display" on the main interface panel. The predicted (possible) failure mode for one-step ahead or multiple-step ahead is displayed in the "Alarm Information" message box. The performance reliability assessments for one-step and multiple-step are shown in the "Reliability Assessment" sub-panel.

A demonstration is developed to test the HPRP model. In order to compare the results of analytical $(\mathrm{Lu}, \mathrm{Lu}$, and Kolarik, 2001) and fuzzy performance reliability models, both models are used in a human task related demonstration. In the demonstration, operators are required to perform a task in a predefined area. Any activities beyond the area are defined as failures. There are two failure modes defined in this demonstration. Failure mode 1 occurs when the position of the hand is too close to a specified point $\left(x_{0}, y_{0}\right)$, falling in a specific zone defined by circle 1 , shown in Figure 5 . Failure mode 2 occurs when the hand is too far from the specific point $\left(x_{0}, y_{0}\right)$ defined by circle 2, shown in Figure 5. As depicted in Figure 5, system failure for this example would result from either a mode 1 failure or a mode 2 failure. In this demonstration, the positions of a sensor in an $x-y$ coordinate system are identified as performance matrices and monitored in real-time by an ISOTRAK II tracking system. The $x$ and $y$ coordinates and reliability estimates are viewed on the computer monitor in real-time and are also stored in the computer for off-line analysis. Reliability for this task is defined as the probability, given the current and past position of the sensor, that it will be positioned within the outer boundary and outside of the inner boundary at a specified future time.

The demonstration compares two performance reliability assessment methods: analytical and fuzzy performance reliability.

\subsection{Analytical performance reliability method}

In the analytical performance reliability assessment, conditional performance is obtained through multi-dimensional integration using Equation (2). According to the task specification and failure definitions in the above paragraph, critical limits regarding failure mode 1 (the position of the operator's hand too close to point $(x 0, y 0))$ are expressed as:

$$
\left(y-y_{0}\right)^{2}+\left(x-x_{0}\right)^{2} \leq c_{1}^{2}
$$

where $c_{1}$ is a predefined distance defined by operational requirements.

Likewise the critical failure mode 2 (the position of the operator's hand too far from point $\left.\left(x_{0}, y_{0}\right)\right)$ can be ex- 


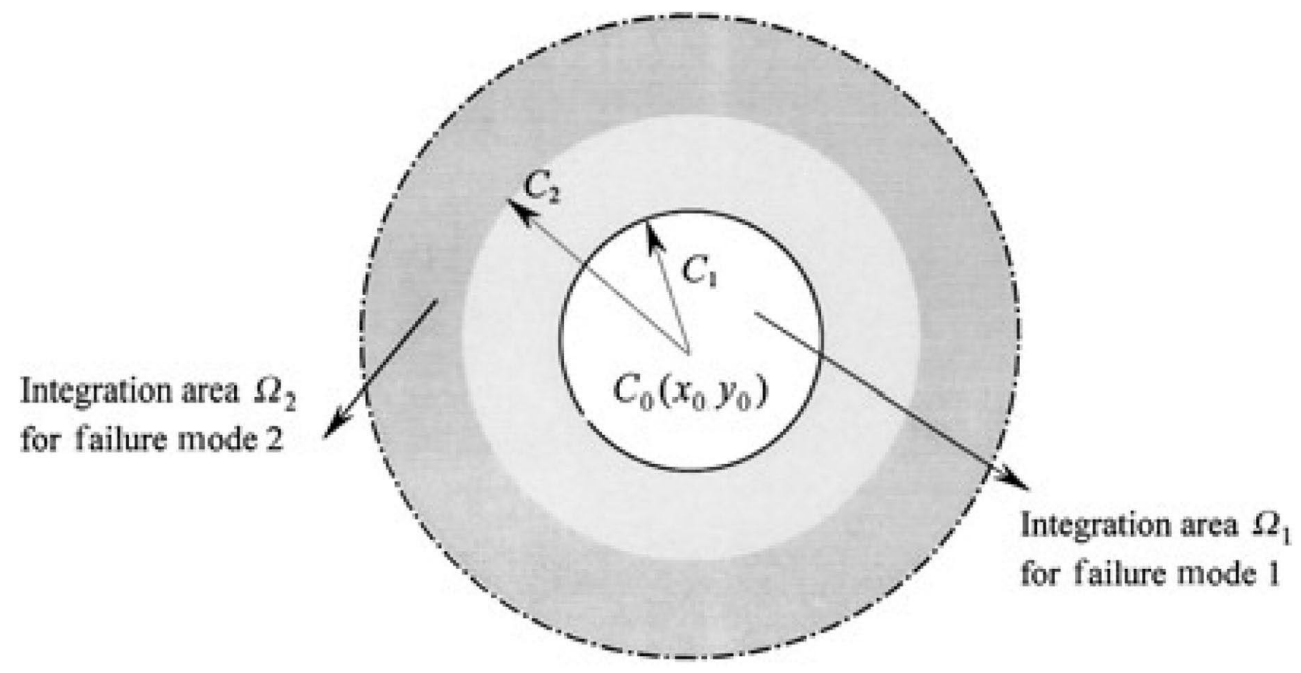

Figure 5. Definition of system failure mode.

pressed as:

$$
\left(y-y_{0}\right)^{2}+\left(x-x_{0}\right)^{2} \geq c_{2}^{2} .
$$

The performance reliabilities regarding these two failure modes are obtained by numerical, multi-dimensional integration over the area defined by Equations (18) and (19). The performance reliability result corresponding to this analytical method is shown in a previous paper $(\mathrm{Lu}$, Lu, and Kolarik, 2001).

\subsection{Fuzzy performance reliability assessment}

Performance reliability is estimated by a fuzzy estimator that is established based on linguistic descriptions of human performance reliability, regarding the performance measures. In this demonstration, there are two performance variables in a crisp domain serving as the inputs of the estimator. In the first step, the performance measures in crisp values are mapped into a universe of discourse of $[-4,4]$ by normalization. Then the normalized values are converted to corresponding fuzzy values by fuzzification. In this demonstration, the variables (two performance measures and performance reliability itself) are described by five fuzzy subsets: very small (0), small (1), medium (2), large (3), and very large (4). Please note that a crisp value can be interpreted as different fuzzy subsets with different membership functions. For example, shown in Figure 6 (Hines, 1997), the normalized value -2.5 is converted to the fuzzy subset "very small" with membership function $\mu_{2}$ or to fuzzy subset "small" with membership function $\mu_{1}$. In general, the more fuzzy subsets defined, the more accurate the output of the fuzzy estimator. However, as the number of subsets increases, more time is required in computation. Therefore, in practice, there is a trade-off between the number of fuzzy subsets defined and timeliness of the estimator.
Corresponding to the five fuzzy subsets for each performance measure used in the demonstration, there are a total of $5 \times 5=25$ fuzzy rules defined. These rules form the reliability algorithm for each failure mode. These rules are constructed from the knowledge about the failures: in this case, the performance reliability regarding failure mode 1 is getting lower when the hand position approaches the point $C_{0}\left(x_{0}, y_{0}\right)$, and the performance reliability regarding failure mode 2 is getting higher when the hand position approaches the point $C_{0}\left(x_{0}, y_{0}\right)$. The following are examples of the 25 fuzzy rules:

Rule 1: If variable $Y$ is medium AND variable $X$ is very small, then performance reliability regarding failure mode 1 is very large, ELSE

Rule 2: If variable $Y$ is medium AND variable $X$ is medium, then performance reliability regarding failure mode 1 is very small, ELSE

Rule 3: If variable $Y$ is medium AND variable $X$ is very large, then performance reliability regarding failure mode 1 is very large, ELSE ...

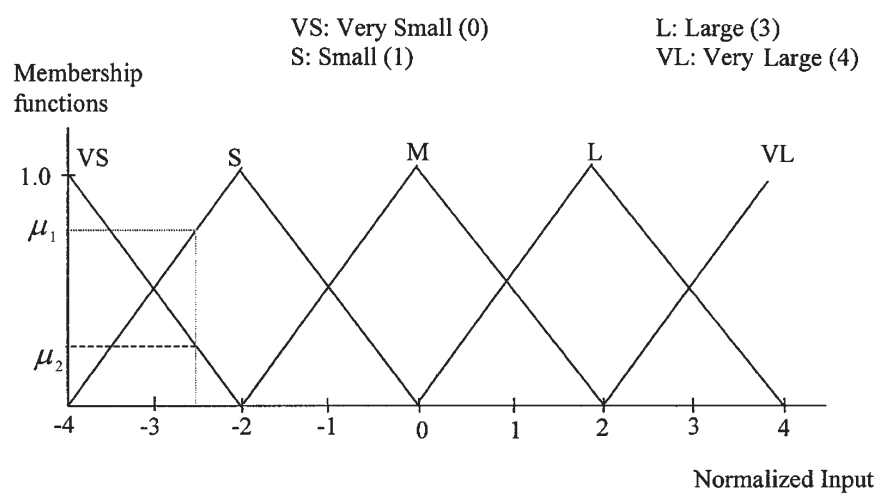

Figure 6. Membership functions of fuzzy sets. 
Table 1. Fuzzy rule table for failure mode 1 in the demonstration of human performance reliability $R_{1}$

\begin{tabular}{lccccc}
\hline \multicolumn{1}{c}{$R_{1}$} & $\begin{array}{c}\text { Very } \\
\text { small (0) }\end{array}$ & $\begin{array}{c}\text { Small } \\
(1)\end{array}$ & $\begin{array}{c}\text { Medium } \\
(2)\end{array}$ & $\begin{array}{c}\text { Large } \\
(3)\end{array}$ & $\begin{array}{r}\text { Very } \\
\text { large (4) }\end{array}$ \\
\hline Very small (0) & 4 & 4 & 4 & 4 & 4 \\
Small (1) & 4 & 3 & 2 & 3 & 4 \\
Medium (2) & 4 & 2 & 0 & 2 & 4 \\
Large (3) & 4 & 3 & 2 & 2 & 4 \\
Very large (4) & 4 & 4 & 4 & 4 & 4 \\
\hline
\end{tabular}

These fuzzy rules are summarized in Tables 1 and 2 . Tables 1 and 2 are fuzzy rules of performance reliability regarding failure mode 1 and failure mode 2, respectively. Based on the above fuzzy rules, multiple fuzzy rules are fired in the fuzzy rule inference, corresponding to performance measures at every instant of time. A DoF value for each rule is calculated by a fuzzy "AND" operation (Hines, 1997):

$$
\operatorname{DoF}=\mu_{\mathrm{A}}\left(V_{1}\right) \wedge \mu_{\mathrm{B}}\left(V_{2}\right)=\min \left(\mu_{\mathrm{A}^{\prime}} \mu_{\mathrm{B}}\right)
$$

Table 2. Fuzzy rule table for failure mode 2 in the demonstration of human performance reliability $R_{2}$

\begin{tabular}{|c|c|c|c|c|c|}
\hline$R_{2}$ & $\begin{array}{c}\text { Very } \\
\text { small (0) }\end{array}$ & $\begin{array}{l}\text { Small } \\
\text { (1) }\end{array}$ & $\begin{array}{l}\text { Medium } \\
\text { (2) }\end{array}$ & $\begin{array}{c}\text { Large } \\
\text { (3) }\end{array}$ & $\begin{array}{r}\text { Very } \\
\text { large (4) }\end{array}$ \\
\hline Very small (0) & 0 & 0 & 0 & 0 & 0 \\
\hline Small (1) & 0 & 2 & 3 & 2 & 0 \\
\hline Medium (2) & 0 & 3 & 4 & 3 & 0 \\
\hline Large (3) & 0 & 2 & 3 & 2 & 0 \\
\hline Very large (4) & 0 & 0 & 0 & 0 & 0 \\
\hline
\end{tabular}

In the above tables, 0 : very small; 1 : small; 2 : medium; 3 : large; 4 : very large.

By defuzzification, all the rules fired in the above step are combined and transferred to a crisp value with the method of "center-of-mass." This method is demonstrated in Figure 7 (Hines, 1997). Here, there are four fuzzy rules fired at the same time. The crisp value is obtained by calculating the gravity center of areas formed by the union of these fired fuzzy rules (Hines, 1997). The formula of
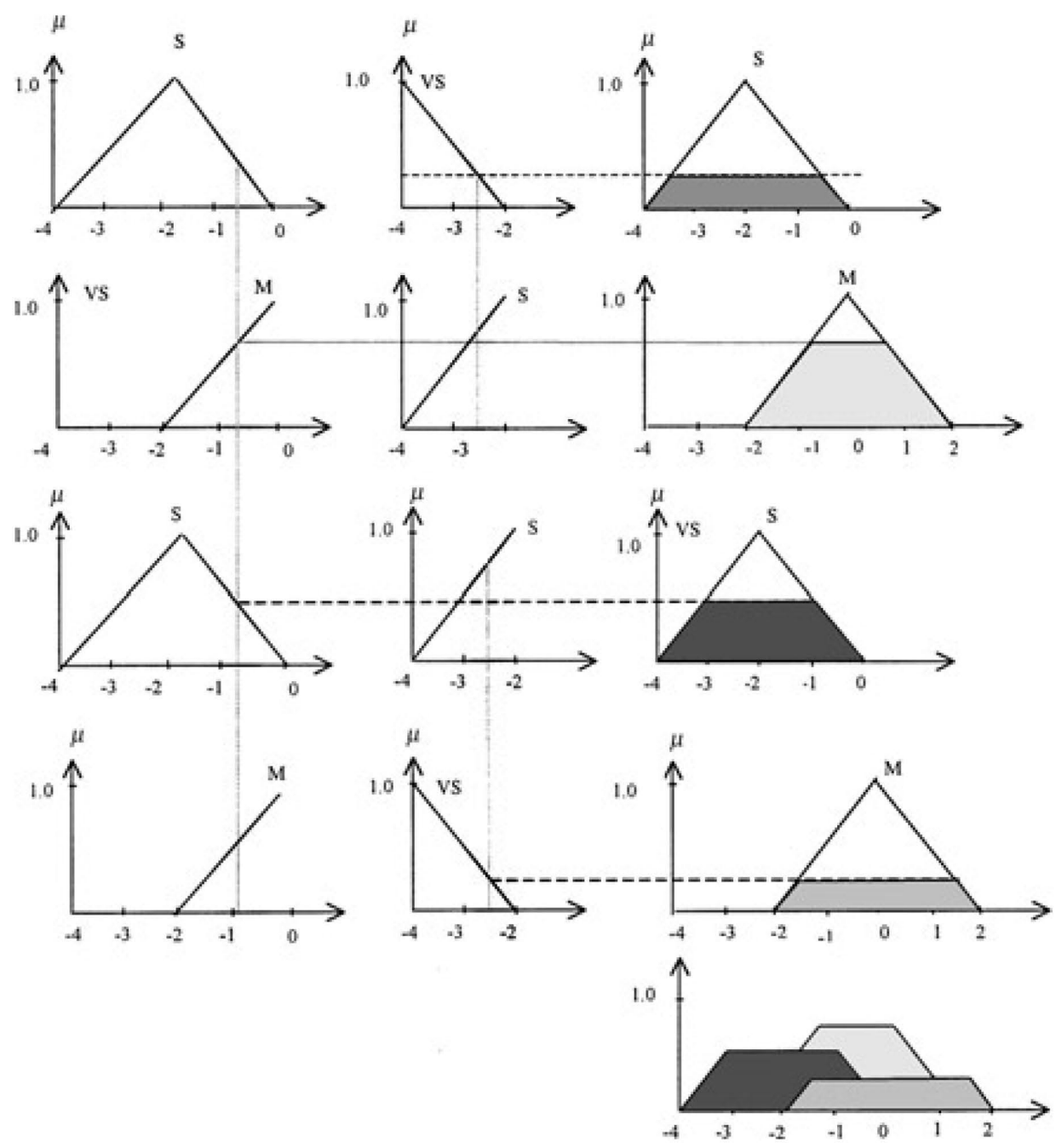

Figure 7. Fuzzy performance reliability assessment at time $t_{0}$. 


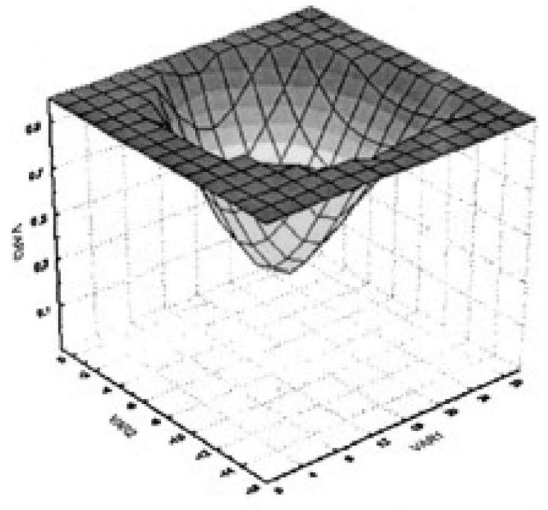

Var1: $x$-position (inch) Var2: $y$-position (inch) Var3: reliability

Figure 8. A three-dimensional plot of the human performance reliability as a function of hand position for failure mode 1 (fuzzy failure definition).

combination/defuzzification is as follows:

$$
\mu=\frac{\sum_{i=1}^{n} \mu_{i} \mu_{\mathrm{out}}\left(\mu_{i}\right)}{\sum_{i=1}^{n} \mu_{\mathrm{out}}\left(\mu_{i}\right)}
$$

In the last step, the crisp value obtained by defuzzification is converted into an original magnitude [0,1], which is the performance reliability output from the fuzzy reliability estimator.

Figures 8-10 show the performance reliability assessment results regarding failure mode 1 , failure mode 2 , and overall failures, based on fuzzy failure estimation. These figures show three-dimensional plots of the human performance reliability as a function of hand position.

A validation test for the model is considered in modeling human performance measurements. The forecast model validation is based on the following measures: Mean- Square Error (MSE), Mean Percentage Error

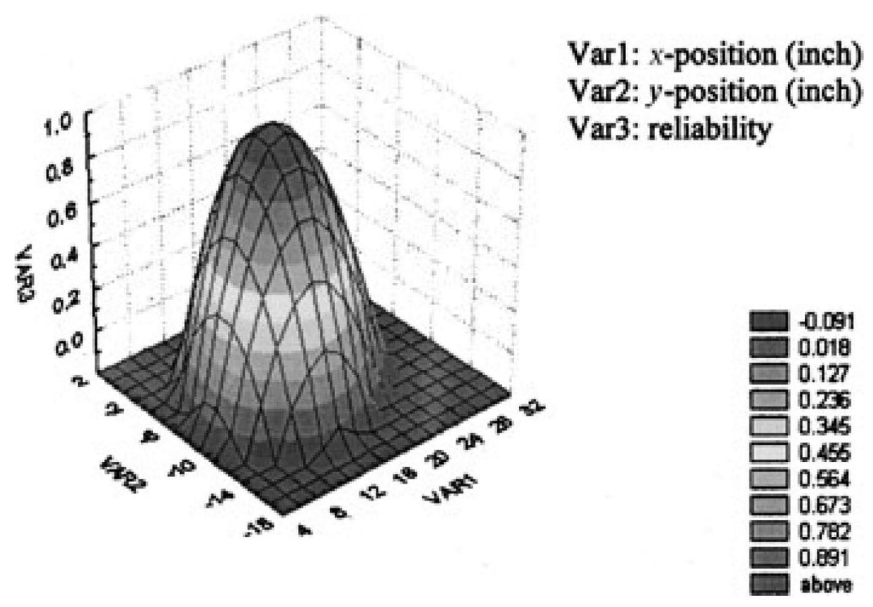

Figure 9. A three-dimensional plot of the human performance reliability as a function of hand position for failure mode 2 (fuzzy failure definition).

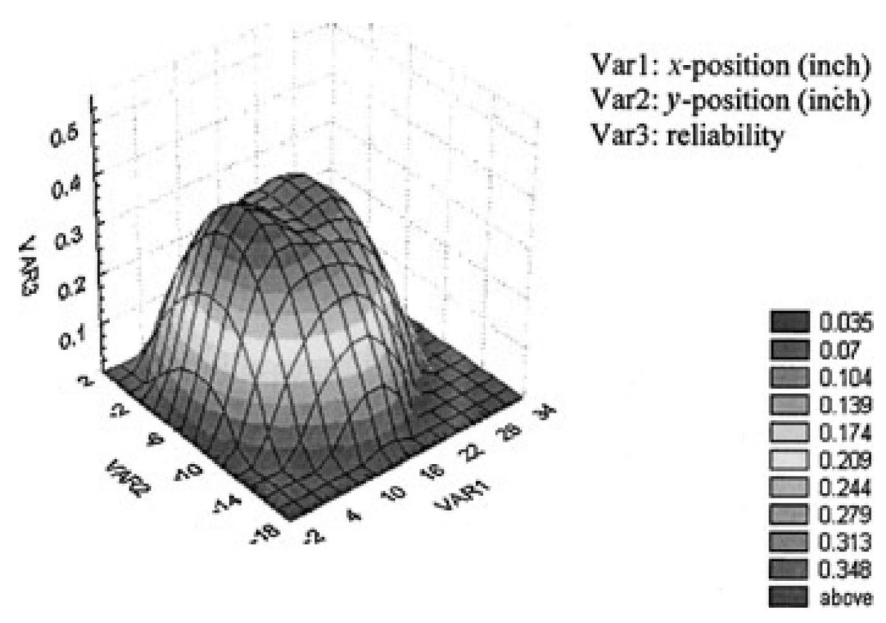

Figure 10. A three-dimensional plot of the human performance reliability as a function of hand position for all failure modes (fuzzy failure definition).

(MPE), Root-Mean-Square Prediction Error (RMSPE), and a white noise test for the forecasting errors. The white noise test is based on the Portmanteau or $Q$ test. If the series $\left\{Y_{t}\right\}$ is from a white process, then the statistic (Brockwell and Davis, 1991):

$$
Q=n(n+2) \sum_{j=1}^{m} \frac{1}{n-j} \hat{\rho}^{2}(j) \rightarrow \chi_{m}^{2}
$$

The hypothesis of white noise is rejected if $Q>x_{1-\alpha, m}^{2}$.

The human performance reliability prediction model is validated based on MSE, MPE, RMSPE, and the white noise test. A comparison of results between the analytical failure definition ( $\mathrm{Lu}, \mathrm{Lu}$, and Kolarik, 2001) and fuzzy failure definition was also conducted (Lu, 1999). The comparison results show that these two methods can produce similar results, as long as the fuzzy rules are defined appropriately.

A fuzzy performance reliability model is a better choice than a crisp performance reliability model in a complex system, where performance measures are difficult to measure precisely and/or the relationship between performance measures and failure modes cannot be represented through analytical models. Fuzzy performance reliability also provides an alternative in the case where there are many performance measures to deal with and numerical integration methods cannot meet timeliness requirements for real-time implementation. Results show that the fuzzy performance model has advantages in simplicity and timeliness, and is capable of satisfying basic accuracy requirements. It appears to be a promising method, capable of accommodating the uncertainty and subjectivity associated with predicting human performance reliability. 


\section{Conclusions}

This paper addresses a real-time HPRP model that is suited to monitoring and predicting an individual's performance when performing a task. Successful implementation of the HPRP model primarily depends on suitable performance measure identification, performance forecasting and failure definitions. Two multivariate forecasting methods, multivariate exponential smoothing and state-space modeling, can be used for performance measurement forecasting. Two failure definition methods, analytical failure definition and fuzzy failure definition, can be used in failure mode definition.

Analytical failure definition is preferred when highaccuracy assessment is needed. Fuzzy performance reliability estimators serve as alternative methods: with advantages in simplicity and timeliness. For example, the exponential smoothing forecasting method and fuzzy failure definition are a possible combination when timeliness is critical in the assessment. In such a situation, one may be forced to make a trade-off between timeliness and accuracy. Fuzzy logic in the reliability estimator, as opposed to analytical methods, allows flexibility in this trade-off.

\section{References}

Akaike, H. (1974) Stochastic theory of minimal realization, IEEE Trans. on Automatic Control, AC-19, 667-674.

Brockwell, P. J. and Davis, R. A. (1991) Time Series: Theory and Methods, 2nd edn., Springer-Verlag, New York, NY.

Driankov, D., Hellendoorn, H., and Reinfrank, M. (1999) An Introduction to Fuzzy Control, 2nd edn., SpringerVerlag, Berlin, Germany.

Embrey, D. E., Humphreys, P. C., Rosa, E. A., Kirvan, B., and Rea, K. (1984) An approach to assessing human error probabilities using structured expert judgment. NUREG/CR-3518 (BNL-NUREG51716), Department of Nuclear Energy, Brookhaven National Laboratory, Upton, NY 11973.

Enn, P. G., Machak, J. A., Spivey, W. A., and Wrobleski, W. J. (1982) Forecasting application of an adaptive multiple exponential smoothing model. Management Science, 28, 1035-1044.

Harvey, A. C. (1990) Estimation procedures for structural time series models. Journal of Forecasting, 9, 89-108.

Hines, J. W. (1997) Fuzzy and Neural Approaches in Engineering, Wiley, New York, NY.
Hannaman, G. W., Spurgin, A. J., and Luckic, Y. D. (1985) A model for assessing human cognitive reliability in PRA studies, in Proceedings of the IEEE Conference on Human Factors and Power Plants, 343- 353.

Kolarik, W. J. (1995) Creating Quality: Concepts, Systems, Strategies, and Tools, McGraw-Hill, New York, NY.

Kolarik, W. J., Lu, H., and Lu, S. (1998) A real-time reliability monitoring prototype, in IIE Research 98 Conference Proceedings, CD IERC, Banff, Canada.

Kolarik, W. J., Woldstad, J., Lu, H., and Lu, S. (1998) New concepts in human ..., CD, 11-14 Oct., San Diego, CA, USA.

Kolarik, W. J., Woldstad, J., and Lu, S. (2000) Real-time human performance reliability assessment, presented at the International Ergonomics Association XIVth Triennial Congress and Human Factors and Ergonomics Society, 44th Annual Meeting, San Diego, CA.

Lu, H. (1998) System self-assessment of survival in time series modeling. Ph.D. Dissertation, Texas Tech University, Lubbock, TX.

Lu, H., Kolarik, W. J., and Lu, S. (2001) Real-time performance reliability prediction. IEEE Transactions on Reliability, 50, 353-357.

Lu, S. (1999) System performance reliability prediction in multivariate time series modeling. Ph.D. Dissertation, Texas Tech University, Lubbock, TX.

Lu, S., Lu, H., and Kolarik, W. J. (2001) Multivariate performance reliability prediction in real-time. Journal of Reliability Engineering and System Safety, 72, 39-45.

Ng, C. N. and Young, P. C. (1990) Recursive estimation and forecasting of non-stationary time series. Journal of Forecasting, 9, 173- 204.

Ogata, K. (1997) Modern Control Engineering, 3rd edn., Prentice-Hall, Upper Saddle River, NJ.

Swain, A. D. and Guttmann, H. E. (1983) Handbook of human reliability analysis with emphasis on nuclear power plant applications. NUREG/CR-1278, US Nuclear Regulatory Commission, Washington, DC.

Weston, L. M., Whitehead, D., and Graves, N. (1987) Recovery actions in PRA for the risk methods integration and evaluation program (RMIEP). NUREG/CR-4834, 1, US Nuclear Regulatory Commission, Washington, DC.

Young, P. C., Ng, C. N., Lane, K., and Parker, D. (1991) Recursive forecasting, smoothing and seasonal adjustment of non-stationary environmental data. Journal of Forecasting, 10, 57-89.

Young, P. C. (1994) Time-variable parameter and trend estimation non- stationary economic time series. Journal of Forecasting, 13, 179- 210. 


\section{The Authors:}

William J. Kolarik is Professor and Head of the School of Industrial Engineering and Management at Oklahoma State University. He holds B.S.I.E. and M.S.I.E. degrees from Louisiana State University and a Ph.D. from Oklahoma State University. He served on the IE faculty at Texas Tech University for over 15 years. He has researched quality and reliability related topics for about two decades and published the results in numerous technical papers. His current research interests lie in the areas of real-time reliability modeling and process definition, design, control, and improvement. He is a Fellow of IIE, a registered professional engineer, and has served as a Baldrige examiner.

Jeffrey C. Woldstad is an Associate Professor of Industrial Engineering at Texas Tech University. He received a B.S. degree in Mathematics and Psychology from the University of Puget Sound in Tacoma, Washington, in 1983, a M.S. degree in Industrial and Operations Engineering from the University of Michigan in Ann Arbor, Michigan, in 1984, and a Ph.D. in Industrial and Operations Engineering and Psychology in 1989, also from the University of Michigan. He has served on the faculty at Oregon State University and at Virginia Polytechnic Institute and State University (Virginia Tech). He is an experienced researcher and educator with expertise in the areas of occupational biomechanics, human motor control, psychophysics, and human information processing. His current research interests include biomechanical evaluations of slip and fall accidents, human reliability and mistake proofing in manufacturing operations, computer modeling of human posture, and motion and biomechanical modeling of neck and shoulder injuries.
Susan Lu is an Assistant Professor of Systems Science and Industrial Engineering, SUNY Binghamton, NY. She received her Ph.D. from the Department of Industrial Engineering at Texas Tech University in 1999. Her current research interests include reliability modeling, survival assessment, process control, and computer integrated manufacturing. She is a member of IIE and Alpha Pi Mu.

Huitian Lu is an Associate Professor in the Department of Engineering Technology and Management, South Dakota State University. He received his M.S. and Ph.D. degrees from Texas Tech University in 1992 and 1998, respectively. He has over 4 years of quality control work experience in semiconductor wafer manufacturing. His current research interests include reliability modeling, design for reliability, intelligent manufacturing, and information systems. He is a member of IIE, SME, and Alpha Pi Mu. 